\title{
Expansão de Lesão por Manchas Foliares em Cevada e sua Interação com a Aplicação Foliar de Fungicidas*
}

\author{
Ana P. Menegon ${ }^{1}$, Carlos A. Forcelini ${ }^{1}$ \& José M.C. Fernandes ${ }^{2}$ \\ ${ }^{1}$ Faculdade de Agronomia e Medicina Veterinária, Universidade de Passo Fundo, Cx. Postal 611, CEP 99001-970, Passo

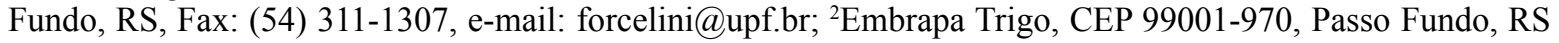

(Aceito para publicação em 01/02/2005)

Autor para correspondência: Carlos Alberto Forcelini

MENEGON, A.P., FORCELINI, C.A. \& FERNANDES, J.M.C. Expansão de lesão por manchas foliares em cevada e sua interação com a aplicação foliar de fungicidas. Fitopatologia Brasileira 30:134-138. 2005.

\section{RESUMO}

A mancha-marrom (MM) e a mancha-reticular (MR), causadas pelos fungos Bipolaris sorokiniana e Pyrenophora teres, respectivamente, são as principais doenças foliares da cevada (Hordeum vulgare). A taxa de expansão da lesão pode ser um componente importante do progresso destas doenças. Em casa de vegetação e em campo, avaliou-se a expansão de lesão em cultivares de cevada, diante da aplicação preventiva ou curativa de um fungicida triazol (ciproconazole + propiconazole) e uma estrobilurina (azoxistrobim). O tamanho médio das lesões (MM) aumentou de 2,2 a $4,9 \mathrm{~mm}^{2}, \mathrm{com}^{2}$ maior incremento entre 14 e 30 dias após a inoculação. As cultivares MN 698 e EMB 128 apresentaram taxas de expansão de lesão maiores que os demais. O tratamento preventivo com fungicida protegeu as plantas de infecção por B. sorokiniana por até 21 dias. Aplicações curativas, realizadas um, dois, quatro, seis, oito, dez ou 12 dias após a inoculação, determinaram lesões iniciais maiores (até $0,68 \mathrm{~mm}^{2}$ ) e mais numerosas (até 3,9 por folha), com ação apenas sobre a esporulação do fungo. Em campo, somente as primeiras aplicações, iniciadas entre 46 e 60 dias após a emergência das plantas, reduziram o tamanho das lesões de MR e a área abaixo da curva de progresso da doença (AACPD), com aumento no rendimento de grãos. Como os fungicidas apresentaram pouco ou nenhum efeito curativo sobre a expansão da lesão, sugere-se reavaliar as recomendações de controle baseadas em severidade de doença.

Palavras-chave adicionais: Bipolaris sorokiniana, Pyrenophora teres, Hordeum vulgare, controle, epidemiologia.

\begin{abstract}
Lesion expansion by barley leaf spots and its interaction with fungicidal sprays

Brown spot and net blotch, caused by the fungi Bipolaris sorokiniana and Pyrenophora teres, respectively, are the main leaf spots of barley (Hordeum vulgare). The rate of lesion expansion is an important epidemiological component of leaf spots and should be taken into account for disease management. Through greenhouse and field trials, lesion expansion was assessed in barley cultivars, under preventive and curative sprays of triazol (cyproconazole + propiconazole) and strobylurin (azoxystrobin) fungicides. In the greenhouse, the average area of brown spot lesions increased from 2.2 to $4.9 \mathrm{~mm}^{2}$, most of which occurred between 14 and 30 days after inoculation. The cultivars MN 698 and EMB 128 showed a higher rate of lesion expansion than BR 2, EMB 127, EMB 129, and MN 684. A single preventive spray of fungicide averted infection by $B$. sorokiniana for over 21 days. In contrast, curative sprays carried out at one, two, four, six, eight, ten, or 12 days after inoculation, although inhibiting fungus sporulation, also resulted in initial lesions that were larger (up to $0.68 \mathrm{~mm}^{2}$ ) and more numerous (up to 3.9 per leaf). In the field, only the spray programs initiated at 46 to 60 days after plant emergence were able to reduce lesion size and the area under the disease progress curve (AACPD) for net blotch, which increased grain yield. Because fungicides had little or no curative effect on lesion expansion, fungicidal sprays based on disease severity should be reevaluated.
\end{abstract}

Additional keywords: Bipolaris sorokiniana, Pyrenophora teres, Hordeum vulgare, control, epidemiology.

\section{INTRODUÇÃO}

Com uma área de 122.000 ha, localizada, principalmente, no Rio Grande do Sul, Santa Catarina e Paraná, a cevada (Hordeum vulgare L.) é um dos principais cereais de inverno cultivados no Brasil (Comissão, 2001). As regiões de cultivo apresentam clima sub-temperado, com chuvas freqüentes e temperaturas médias de 12 e $22{ }^{\circ} \mathrm{C}$, nos meses mais frios e mais quentes, respectivamente. Tais condições

\footnotetext{
*Parte da Dissertação de Mestrado do primeiro autor, Universidade de Passo Fundo (2003).
}

climáticas favorecem a ocorrência de doenças fúngicas (Minella et al., 1981).

As duas principais doenças foliares da cevada são a mancha-reticular (ou mancha reticular) e a mancha-marrom, causadas, respectivamente, pelos fungos Pyrenophora teres (Sacc.) Shoem. e Bipolaris sorokiniana (Sacc. In. Sorok.) Shoem. (Luz, 1982; Forcelini \& Reis, 1997; Mathre, 1997). Em cultivares suscetíveis, o dano médio atribuído a estas doenças varia de 10 a $40 \%$ para a mancha-reticular (Mathre, 1997) e de 16 a 33\% para a mancha-marrom (Clark, 1979; Wilcoxson et al., 1990), embora estes sejam maiores quando 
Expansão de lesão por manchas foliares em cevada e...

as doenças iniciam antes do estádio de emborrachamento (Nutter et al., 1985).

O controle das manchas foliares da cevada inclui a rotação de culturas, o uso de cultivares resistentes, a utilização de sementes sadias ou tratadas e a aplicação foliar de fungicidas. Esta última é recomendada a partir do estádio de alongamento, quando a severidade das manchas foliares atinge 5\% (Comissão, 2001).

O progresso de uma doença pode ser explicado pelo aparecimento de novas lesões e pelo crescimento daquelas já existentes (Bergamin Filho \& Amorim, 1996). A expansão da lesão reflete a ação infecciosa do patógeno, enquanto novas infecções somente o fazem após um período de latência (Berger et al., 1997). Assim, a taxa de expansão da lesão é um componente importante no processo epidêmico.

O tamanho da lesão e a taxa de expansão têm sido utilizados para determinar a resistência genética de plantas, bem como, avaliar a agressividade de estirpes ou raças de bactérias e fungos fitopatogênicos (Johnson \& Taylor, 1976; Luo \& Zeng, 1995; Berger et al., 1997). Em trigo (Triticum aestivum L.), Mehta (1981) verificou que uma simples lesão da mancha-marrom afetou $23 \%$ da área da folha, em um período de 33 dias. Contudo, pouco se sabe sobre como o controle químico influencia o processo de expansão de lesão.

Nos estádios iniciais da mancha-marrom e manchareticular, as lesões são pequenas e, embora numerosas, representam uma severidade baixa, aquém do limiar de ação para controle químico. Contudo, as lesões aumentam de tamanho e podem atingir grandes áreas, comprometendo o controle das doenças e o rendimento da cultura. Por este motivo, torna-se necessário conhecer e quantificar este processo em cevada, assim como verificar como este se relaciona com as diferentes cultivares utilizadas e como interfere no controle químico das manchas foliares.

\section{MATERIAL E MÉTODOS}

Os experimentos foram conduzidos em casa de vegetação (temperatura ambiente entre $20 \mathrm{e} 25^{\circ} \mathrm{C}$ ) e no campo experimental da FAMV/UPF, em 2001 e 2002. Inicialmente, cultivares de cevada (BR 2, EMB 127, EMB 128, EMB 129, MN 684 e MN 698) foram avaliadas quanto à taxa de expansão de lesões da mancha-marrom em casa-de-vegetação. A partir da semeadura em vasos (em 07/05/01), as plantas foram inoculadas com suspensões de esporos (2.500 a 20.000 esporos $/ \mathrm{ml}$ ) de $B$. sorokiniana, aos 30 dias após a semeadura. De cada cultivar, quatro vasos (repetições) contendo plantas com dez a 15 lesões por folha foram selecionados, sendo as lesões marcadas e medidas duas vezes por semana, no período entre seis e 43 dias após a inoculação, através de um paquímetro digital com precisão de $0,01 \mathrm{~mm}$.

Em um segundo experimento estabelecido em casade-vegetação, em 18/03/02, avaliou-se o efeito de aplicações preventivas e curativas de fungicidas no processo de expansão de lesão. Utilizaram-se plantas da cultivar BR 2, aos 45 dias após a semeadura, e os fungicidas azoxistrobim (Priori®, 200 $\mathrm{ml} /$ ha) e propiconazole + ciproconazole (Artea ${ }^{\circledR}, 300 \mathrm{ml} /$ ha), os quais pertencem aos grupos químicos estrobilurina e triazol, respectivamente. Plantas de 42 vasos, 21 para cada fungicida, foram pulverizadas e depois inoculadas com $B$. sorokiniana (5000 esporos $/ \mathrm{ml}$ ) aos três, seis, nove, 12, 15, 18 e 21 dias após o tratamento químico. Outro grupo de plantas, em 42 vasos, foi inoculado com a mesma quantidade de esporos e após um, dois, quatro, seis, oito, dez e 12 dias pulverizado com os fungicidas. A pulverização foi realizada com o mesmo equipamento e ajustes utilizados em campo, conforme descrito a seguir. As plantas foram avaliadas quanto ao número e tamanho das lesões, em intervalos de dois-três dias, em folhas previamente marcadas. Em cada avaliação, folhas com lesões foram retiradas das plantas, incubadas em câmara úmida a $25^{\circ} \mathrm{C}$, por três dias, e examinadas quanto à esporulação de $B$. sorokiniana sobre as lesões. Nestes dois experimentos, foram utilizadas três repetições (vasos), cada qual com cinco plantas, para cada tratamento.

Em campo, 36 parcelas $(1 \times 5 \mathrm{~m})$ da cultivar MN 698, estabelecidas em 30/06/01, de sementes tratadas com o fungicida triadimenol (Baytan ${ }^{\circledR}, 270 \mathrm{ml} / 100 \mathrm{~kg}$ ) e o inseticida imidacloprid (Gaucho®, $100 \mathrm{~g} / 100 \mathrm{~kg}$ ), foram utilizadas para avaliar o efeito da época de aplicação foliar de fungicidas na expansão das lesões, no controle das manchas foliares e no rendimento de grãos. O número de repetições foi igual a três. Utilizaram-se os mesmos fungicidas mencionados anteriormente, em tratamentos iniciados aos 46, 53, 60, 67 e 74 dias após a emergência e repetidos uma vez, com um intervalo de 21 dias. O volume de calda foi de $150 \mathrm{l} / \mathrm{ha}$, aplicado através de pontas do tipo leque (XR 11015), em equipamento pressurizado com $\mathrm{CO}_{2}$.

Em sete intervalos semanais $(23 / 08,30 / 08,06 / 09,13 /$ 09, 21/09, 27/09 e 18/10), do estádio final do perfilhamento até o florescimento pleno, procederam-se coletas de 10 plantas por parcela, as quais foram avaliadas quanto à severidade das manchas foliares e ao tamanho das lesões. A severidade foi integralizada como área abaixo da curva de progresso da doença (AACPD), através da fórmula AACPD $=\Sigma\left[\left(y_{1}+\right.\right.$ $\left.\left.y_{2}\right) / 2\right) *\left(t_{2}-t_{1}\right)$ ], onde $y_{1}$ e $y_{2}$ são duas avaliações consecutivas realizadas nos tempos $t_{1}$ e $t_{2}$, respectivamente. A colheita ocorreu em 13/11/01.

Modelos lineares, concomitantes com a análise de variância e o teste de Duncan, foram usados para testar o efeito dos fungicidas e do momento de aplicação sobre o processo de formação e expansão de lesão. Contrastes ortogonais foram utilizados para comparações de tratamentos com a testemunha sem fungicida. As análises foram realizadas utilizando-se o pacote estatístico disponível no aplicativo SAS, versão 6.12 .

\section{RESULTADOS}

O tamanho médio das lesões da mancha-marrom (Tabela 1) aumentou de 2,2 a $4,9 \mathrm{~mm}^{2}$, representando um incremento médio de $0,072 \mathrm{~mm}^{2} /$ dia. A integralização do tamanho médio das lesões totalizou 146,6 unidades. Entre as 
cultivares, houve variação significativa para todos os componentes do processo de expansão, com maior suscetibilidade da 'MN 698' e da 'EMB 128'. Ressalta-se que, à época, 'MN 698' era a cultivar mais utilizada pelos produtores, em função de sua qualidade para produção de malte.

O maior aumento no tamanho da lesão (Figura 1) da cultivar mais suscetível (MN 698) ocorreu entre 14 e 30 dias após a inoculação, com posterior estabilização do crescimento. Neste sentido, aplicações de fungicida visando o controle desta variável epidêmica deveriam ser adotadas antes deste período. Contudo, os experimentos conduzidos em casa-de-vegetação, não evidenciaram efeito significativo de aplicações curativas de fungicidas sobre o tamanho final da lesão $(P=0,19 \mathrm{e}$ $0,41)$ (Figura 3$)$ e sua taxa de progresso diário $(P=0,12 \mathrm{e}$ $0,1)$. Além disso, o número de lesões por folha (Figura 2, com $P$ de 0,0005 para o triazol e 0,0002 para a estrobilurina), e o tamanho inicial das lesões (com $P$ de 0,005 para o triazol e 0,0006 para a estrobilurina), foram maiores à medida que a aplicação de fungicidas variou de um a 12 dias após a inoculação.

Por outro lado, o uso preventivo de ambos os fungicidas protegeu as plantas eficazmente, uma vez que inoculações realizadas até 21 dias após o tratamento não resultaram em infecção visível. Os tratamentos curativos inibiram a esporulação de $B$. sorokiniana sobre as folhas.

No experimento a campo, houve predominância da mancha-reticular, seguida pelo oídio causado por Blumeria graminis f.sp. tritici (D.C.) E.O. Speer, com ocorrência mínima da mancha-marrom. O tamanho médio das lesões da mancha-reticular (Figura 4) foi menor ( $P$ de 0,041 para o triazol e 0,035 para a estrobilurina) nas plantas tratadas a partir de 46 e 53 dias após a emergência, que diferiram da testemunha por contrastes ortogonais. A reduzida variação observada indica um pequeno efeito do tratamento sobre este componente da epidemia. Possivelmente, o controle da doença pelos fungicidas ocorreu mais pela inibição de novas infecções.

Apenas as duas primeiras épocas de aplicação

TABELA 1 - Tamanho médio de lesões da mancha-marrom, causada por Bipolaris sorokiniana, em plantas de cevada (Hordeum vulgare) de seis cultivares inoculadas aos 30 dias após a semeadura, em casa de vegetação. FAMV/UPF, 2001

\begin{tabular}{lcccc}
\hline \hline \multirow{2}{*}{ Cultivar } & \multicolumn{3}{c}{ Tamanho da les ão $\left(\mathbf{m m}^{\mathbf{2}}\right)$} & \multirow{2}{\text{Taxa}}{} \\
\cline { 2 - 4 } & Inicial & Final & Integral $^{\mathbf{1}}$ & $\left.\mathbf{m m}^{\mathbf{2}} / \mathbf{d i a}\right)$ \\
\hline MN 698 & $3,1 \mathrm{a}^{2}$ & $6,7 \mathrm{a}^{2}$ & $179,6 \mathrm{a}^{2}$ & $0,097 \mathrm{a}^{2}$ \\
EMB 128 & $3,1 \mathrm{a}$ & $5,8 \mathrm{ab}$ & $178,0 \mathrm{a}$ & $0,073 \mathrm{ab}$ \\
EMB 129 & $1,9 \mathrm{~b}$ & $4,6 \mathrm{c}$ & $138,8 \mathrm{~b}$ & $0,073 \mathrm{ab}$ \\
BR 2 & $2,1 \mathrm{~b}$ & $4,9 \mathrm{bc}$ & $131,5 \mathrm{~b}$ & $0,076 \mathrm{ab}$ \\
MN 684 & $1,3 \mathrm{c}$ & $3,6 \mathrm{~d}$ & $126,1 \mathrm{~b}$ & $0,062 \mathrm{~b}$ \\
EMB 127 & $2,0 \mathrm{~b}$ & $3,9 \mathrm{~cd}$ & $125,5 \mathrm{~b}$ & $0,051 \mathrm{~b}$ \\
\hline Média & 2,2 & 4,9 & 146,6 & 0,072 \\
\hline CV (\%) & 7,3 & 6,8 & 11,6 & 12,5 \\
\hline
\end{tabular}

${ }^{1}$ Integralizada pela fórmula $Y=\Sigma\left(\left(\left(x_{1}+x_{2}\right) / 2\right) \times\left(t_{2}-t_{1}\right)\right)$, onde $x_{1}$ e $x_{2}$ são duas medições sucessivas realizadas nos tempos $t_{1}$ e $t_{2}$, respectivamente;

${ }^{2}$ Médias seguidas pela mesma letra não diferem entre si pelo teste de Duncan a $5 \%$ de significância.

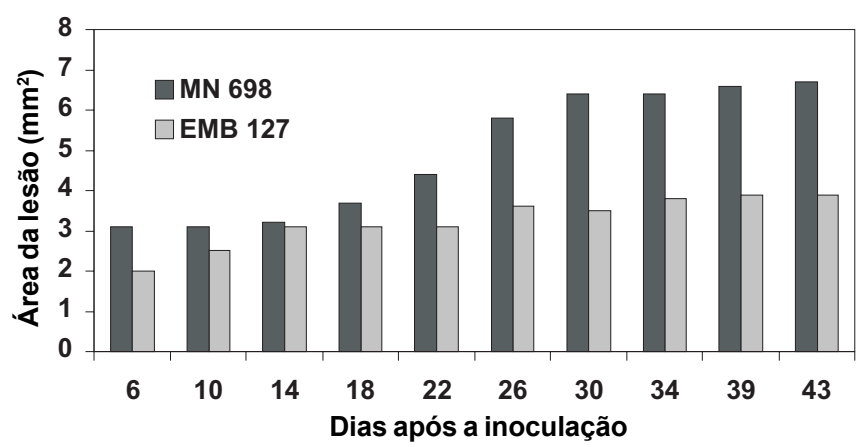

FIG. 1 - Evolução na área da lesão $\left(\mathrm{mm}^{2}\right)$ da mancha-marrom da cevada (Hordeum vulgare), causada por Bipolaris sorokiniana, em duas cultivares, entre seis e 43 dias após a inoculação. FAMV/UPF, 2001 .

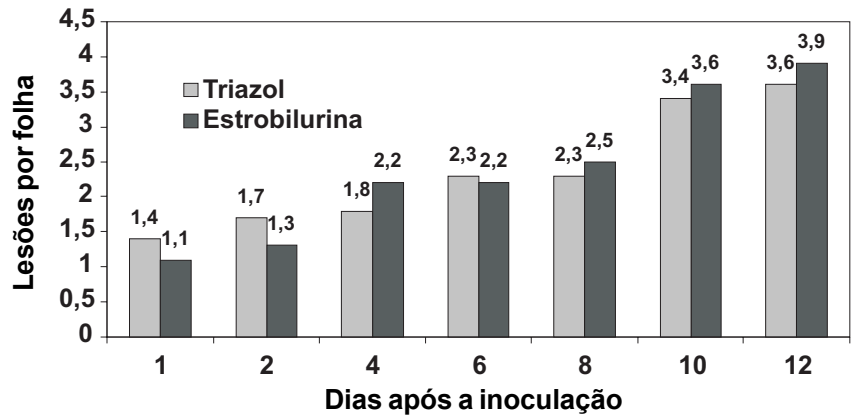

FIG. 2 - Número de lesões por folha da mancha-marrom da cevada (Hordeum vulgare), causada por Bipolaris sorokiniana, após a aplicação de um fungicida triazol (propiconazole + ciproconazole) ou estrobilurina (azoxistrobim), a um, dois, quatro, seis, oito, dez ou 12 dias depois da inoculação. Variação significativa por análise de regressão. FAMV/UPF, 2001.

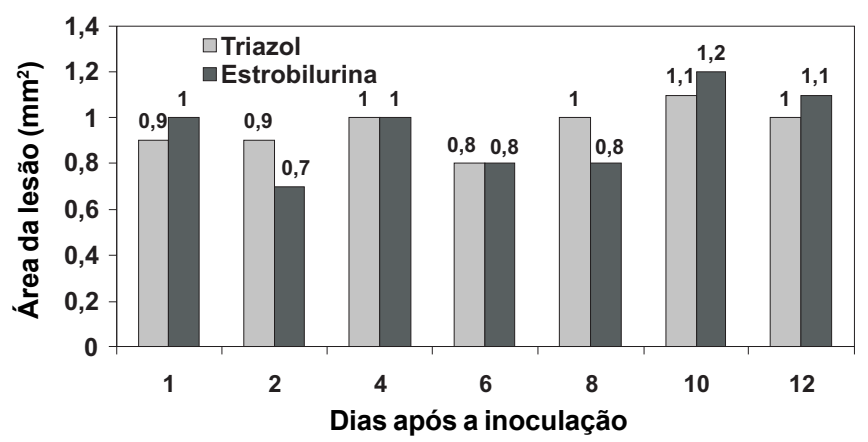

FIG. 3 - Área final $\left(\mathrm{mm}^{2}\right)$ de lesões da mancha-marrom da cevada (Hordeum vulgare), causada por Bipolaris sorokiniana, após a aplicação de um fungicida triazol (propiconazole + ciproconazole) ou estrobilurina (azoxistrobim), a um, dois, quatro, seis, oito, dez ou 12 dias depois da inoculação. Variação não significativa por análise de regressão. FAMV/UPF, 2001.

reduziram, significativamente $(P$ de 0,022 para o triazol e 0,028 para a estrobilurina), a AACPD (Figura 5) em relação às testemunhas sem fungicida. Em relação aos fungicidas, a 
mistura de propiconazole + ciproconazole apresentou maior eficácia que o azoxistrobim sozinho.

O rendimento de grãos (Figura 6) foi parcialmente afetado por chuva de granizo ocorrida em $09 / 09 / 01$, por isso os baixos valores obtidos. Nas áreas sem tratamento, a produtividade variou de 1262 a $1351 \mathrm{~kg} / \mathrm{ha}$, sendo maior à medida que as aplicações foram iniciadas mais cedo $(P$ de 0,027 para o triazol e 0,029 para a estrobilurina). Verificouse maior rendimento nas parcelas tratadas com o fungicida triazol, possivelmente, em função de um melhor controle do oídio, também presente nas plantas. Em geral, as aplicações de fungicida iniciadas mais tarde, em 12/09 (4a época) e 19/ 09 ( $5^{\mathrm{a}}$ época), apresentaram pouco ou nenhum efeito sobre a doença e o rendimento de grãos.

\section{DISCUSSÃO}

Em estudo sobre a expansão de lesão por manchas foliares na cultura do trigo, Prates \& Fernandes (2001) verificaram que este processo, assim como a suscetibilidade de cultivares à mancha foliar causada por B. sorokiniana, são influenciados pela temperatura. Ambos os fatores foram mais intensos entre 23 e $30^{\circ} \mathrm{C}$ que entre 8 e $15^{\circ} \mathrm{C}$. Em nossos experimentos com cevada, a temperatura no interior da casa de vegetação variou entre 20 e $25^{\circ} \mathrm{C}$, sendo próxima à faixa ideal requerida pelo patógeno. Estas temperaturas também se assemelham à média normal na primavera, período no qual o desenvolvimento das manchas foliares da cevada é mais intenso. Assim, as condições utilizadas foram representativas das que favorecem à ocorrência da doença no campo.

Embora as aplicações curativas de fungicida tenham inibido a esporulação do fungo sobre as lesões, estas continuaram a se expandir. Estes resultados estão em acordo com o que afirmam Bergamin Filho \& Amorim (1996), segundo os quais a expansão de lesão representa uma forma alternativa para aumento da doença em condições desfavoráveis à esporulação. Embora os fungicidas utilizados fossem de dois grupos químicos, com dois mecanismos de ação diferentes, ambos atuaram de forma semelhante sobre as variáveis analisadas (esporulação, lesões novas e expansão da lesão).

Até recentemente, o controle químico das manchas foliares em cevada era recomendado quando a severidade atingisse 5\% (Comissão, 2001). Diante dos resultados obtidos neste trabalho, este limiar de ação é muito elevado. Considerando-se que as lesões iniciais são pequenas, muitas vezes menores que $1 \mathrm{~mm}^{2}$, e que uma folha de cevada pode ter área de 15 a $20 \mathrm{~cm}^{2}$, de 7500 a 10000 lesões seriam necessárias para compor uma área foliar afetada de 5\%. Sabendo-se que estas evoluem significativamente e que o efeito de aplicações curativas sobre a expansão é mínimo, recomendações de controle baseadas em níveis de severidade não representam boa estratégia de manejo das manchas foliares. Resultados semelhantes foram obtidos por Mehta et al. (1978), para doenças foliares do trigo.

A partir dos resultados obtidos, sugere-se que o controle químico seja implementado no início da epidemia, sinalizado

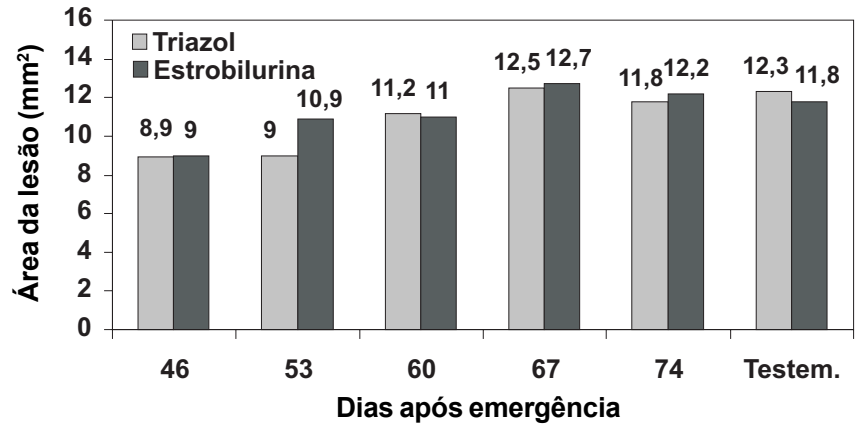

FIG. 4 - Área média $\left(\mathrm{mm}^{2}\right)$ de lesões da mancha-reticular da cevada (Hordeum vulgare), causada por Pyrenophora teres, após aplicação de fungicida triazol (propiconazole + ciproconazole) ou estrobilurina (azoxistrobim). Cada fungicida foi aplicado duas vezes, com intervalo de 21 dias, a partir de 46, 53, 60,67 ou 74 dias após a emergência das plantas. Variação significativa por análise de regressão entre dias e área da lesão. Coeficiente de variação de 18,1\%. FAMV/ UPF, 2001.

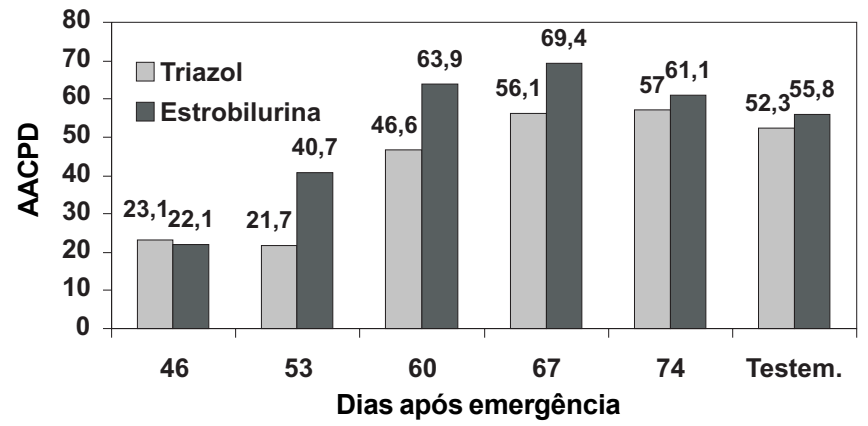

FIG. 5 - Área abaixo da curva de progresso de doença (AACPD) para a mancha-reticular da cevada (Hordeum vulgare), causada por Pyrenophora teres, em função da aplicação de um fungicida triazol (propiconazole + ciproconazole) ou estrobilurina (azoxistrobim). Cada fungicida foi aplicado duas vezes, com intervalo de 21 dias, a partir de 46, 53, 60, 67 ou 74 dias após a emergência das plantas. Variação significativa por análise de regressão entre dias e AACPD. Coeficiente de variação de 21,6\%. FAMV/UPF, 2001.

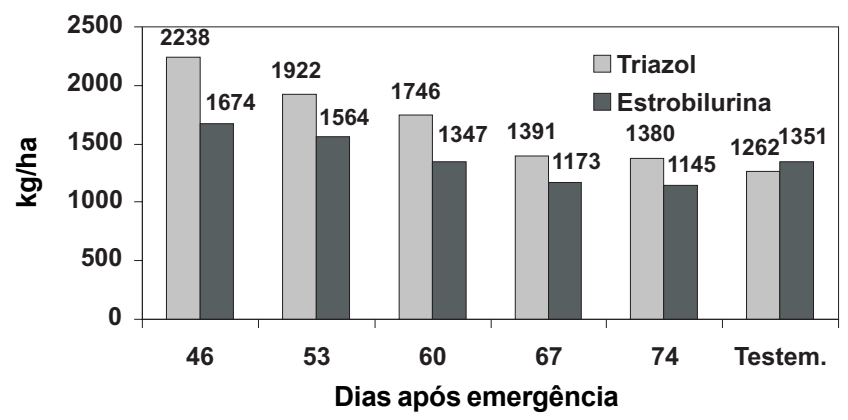

FIG. 6 - Rendimento de grãos (kg/ha) de cevada (Hordeum vulgare) (cv. MN 698) em função da aplicação dos fungicidas propiconazole + ciproconazole (triazol) ou azoxistrobim (estrobilurina). Cada fungicida foi aplicado duas vezes, com intervalo de 21 dias, a partir de 46, 53, 60, 67 ou 74 dias após a emergência das plantas. Variação significativa por análise de regressão entre dias e kg/ha. Coeficiente de variação de 17,4\%. FAMV/UPF, 2001. 


\section{A.P. Menegon et al.}

pelo aparecimento dos primeiros sintomas. Medidas preventivas como a rotação de culturas e o tratamento de sementes de boa qualidade, com fungicidas mais eficazes, auxiliam no manejo destas doenças, retardando o seu aparecimento das manchas foliares e/ou reduzindo sua intensidade inicial.

O reduzido efeito dos fungicidas sobre o processo de expansão das lesões, após o seu estabelecimento na planta, contrasta com a excelente ação preventiva demonstrada por ambos os produtos utilizados e enfatiza a necessidade de controlar as manchas foliares na fase inicial da epidemia. Em um cenário com dois tratamentos ao longo do ciclo da cultura, o primeiro pode ser realizado no aparecimento dos primeiros sintomas e o segundo no estádio de florescimento, quando, tradicionalmente, os produtores procuram conciliar o controle das doenças foliares com as que ocorrem exclusivamente na espiga, como a giberela.

\section{REFERÊNCIAS BIBLIOGRÁFICAS}

BERGAMIN FILHO, A. \& AMORIM, L. Doenças de plantas tropicais: epidemiologia e controle econômico. São Paulo. Ed. Agronômica Ceres. 1996.

BERGER, R.D., BERGAMIN FILHO, A. \& AMORIM, L. Lesion expansion as an epidemic component. Phytopathology 87:10051013. 1997.

CLARK, R.V. Yield losses in barley cultivars caused by spot blotch. Canadian Journal of Plant Pathology 1:113-117. 1979.

COMISSÃO DE PESQUISA DE CEVADA. Indicações técnicas para produção de cevada cervejeira: safras 2001 e 2002. Passo Fundo. Embrapa Trigo. 2001.

FORCELINI, C.A. \& REIS, E.M. Doenças da cevada. In: Kimati, H., Amorim, L., Bergamin Filho, A., Camargo, L.E.A. \& Rezende,
J.A.M. (Eds.) Manual de Fitopatologia. Vol. 2. São Paulo. Ed. Agron. Ceres. 1997. pp. 251-256.

JOHNSON, R. \& TAYLOR, A.J. Spore yield of pathogens in investigations of the race-specificity of host resistance. Annual Review of Phytopathology 14:97-119. 1976.

LUO, Y. \& ZENG, S.M. Simulation studies on epidemics of wheat stripe rust (Puccinia striiformis) on slow-rusting culivars and analysis of effects of resistance components. Plant Pathology 44:340-349. 1995.

LUZ, W.C. da. Diagnose das doenças da cevada no Brasil. Passo Fundo. Embrapa Trigo. Circular Técnica, 2. 1982.

MATHRE, D.E. Compendium of barley diseases. St. Paul. The Americam Phytopathological Society. 1997.

MEHTA, Y.R. Produção de conídios, período de esporulação e extensão da lesão por Helminthosporium sativum nas folhasbandeiras de trigo. Pesquisa Agropecuária Brasileira 16:77-79. 1981.

MEHTA, Y.R., IGARASHI, S. \& NAZARENO, N. Um novo método para avaliar fungicidas contra doenças foliares do trigo. Summa Phytopathologica 4:55-66. 1978.

MINELLA, E., WENDT, W. \& LUZ, W. C. da. Recomendações técnicas para o cultivo da cevada cervejeira. Passo Fundo. Embrapa Trigo. Circular Técnica, 1. 1981.

NUTTER, F.W., PEDERSON Jr, V.D \& FOSTER, A.E. Effect of inoculations with Cochliobolus sativus at specific growth stages on grain yield and quality of malting barley. Crop Science 25:933938. 1985.

PRATES, L.G. \& FERNANDES, J.M.C. Avaliando a taxa de expansão de lesões de Bipolaris sorokiniana em trigo. Fitopatologia Brasileira 26:185-191. 2001.

WILCOXSON, R.D., RASMUSSON, D.C. \& MILES, M.R. Development of barley resistance to spot blotch and genetics of resistance. Plant Disease 74:207-210. 1990. 